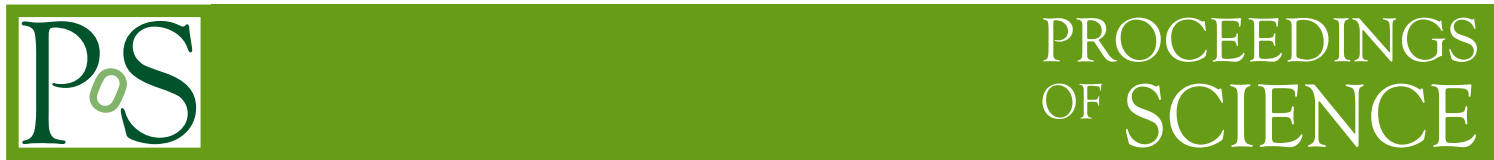

\title{
Universal properties of large $N$ phase transitions in Wilson loops
}

\section{R. Narayanan}

Department of Physics, Florida International University, Miami, FL 33199, USA

E-mail: rajamani.narayananefiu.edu

\section{H. Neuberger*}

Rutgers University, Department of Physics and Astronomy, Piscataway, NJ 08855, USA

E-mail: neuberg@physics.rutgers.edu

Numerical studies support the conjecture that in continuum planar QCD the eigenvalue density of a Wilson loop operator undergoes a transition as the loop is dilated while keeping the loop shape fixed. A second part of the conjecture is that the transition obeys large $N$ universality and that this universality class is the same in 2, 3 and 4 Euclidean space-time dimensions. The focus of the talk will be on clarifying precisely what the conjecture is claiming.

The XXV International Symposium on Lattice Field Theory

July 30-4 August 2007

Regensburg, Germany

${ }^{*}$ Speaker. 


\section{Introduction.}

The main claim of this talk is the following: The eigenvalue distribution associated with a circular, planar Wilson loop at infinite $N$ undergoes a phase transition of universal character as the loop is dilated. The phase transition occurs when the eigenvalue distribution changes from having support on an arc with two distinct endpoints to covering the entire unit circle. The transition persists in the continuum limit for properly regulated Wilson loops, $W$. For these operators, the short distance - long distance cross-over occurs over a scale range that shrinks to zero as $N$ increases [1].

\section{Universality.}

At infinite $N$, for a small loop, the tail of the distribution at eigenvalues close to -1 disappears, and there is a gap. For finite $N$ the tail is suppressed as $e^{-c N}, c>0$. For a large loop there is no gap. The transition happens at a critical scale where it is governed by a large $N$ universality class. The universality class is the same in two, three and four Euclidean dimensions. The two dimensional model is exactly soluble and therefore the universality class is fully understood.

\section{The exactly soluble representative of the large $N$ universality class.}

A general formulation of the universality class [2] is in terms of a random multiplicative ensemble: Consider $n$ independent $S U(N)$ matrices i.i.d. with a measure $d \mu$. The Wilson loop $W$ of unit-less area $t=n \varepsilon^{2}$ is represented by a product of the unitary matrices, in any fixed order, and evolves in $t$ by diffusion on $S U(N)$ starting from a point source. This is exactly true in two dimensions and the relevance of the associated Durhuus-Olesen [3] universality class to higher dimensions is an exact version of the old conjecture of "dimensional reduction".

\section{Average characteristic polynomial.}

The average characteristic polynomial $Q_{N}$ of the fluctuating unitary matrix $W$ at a unit-less loop scale $t$ can be defined in any dimension and captures the essential features of the collective behavior of the $W$ eigenvalues:

$$
Q_{N}(z, t) \equiv\langle\operatorname{det}(z-W(t))\rangle
$$

We are interested in the region around eigenvalue -1 which affects $Q_{N}(z, t)$ mainly around $z=-1$. In two dimensions the exact result is obtained as $\varepsilon \rightarrow 0, n \rightarrow \infty$ at fixed $t$ and is critical at $t=t_{c}=4$.

\section{1 $Q_{N}(z, t)$ in two dimensions.}

In two dimensions we can calculate $Q_{N}(z, t)$ exactly. Applying the Lee-Yang theorem to the result we prove that all the zeros of $Q_{N}(z, t)$ lie on the unit circle. Figure 1 shows this graphically. 


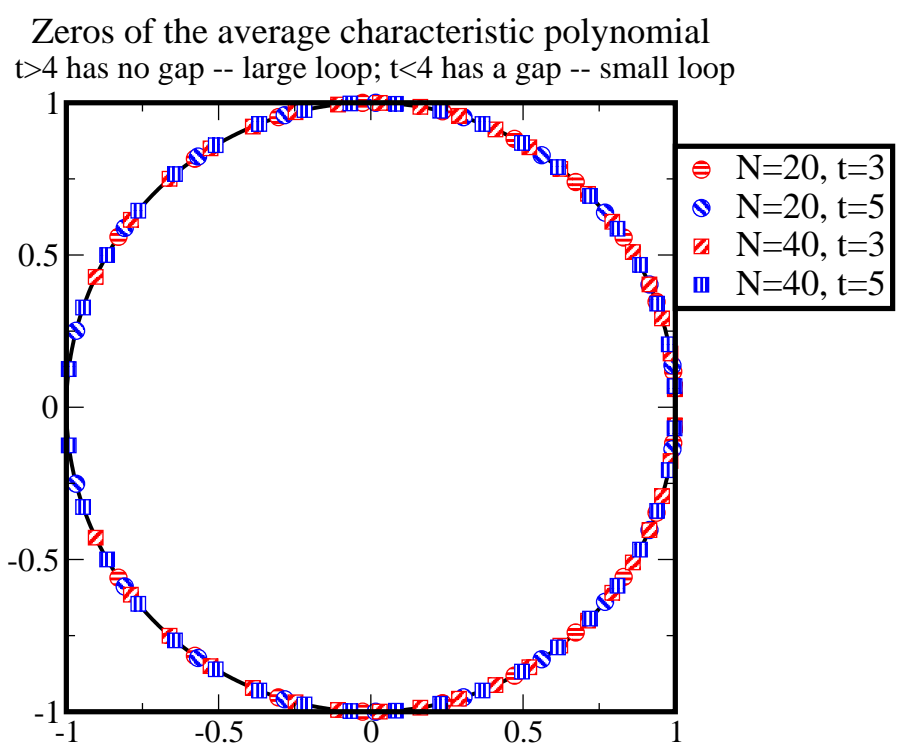

Figure 1: Locations of "charges" in the complex $z$-plane; we are interested in a small arc centered at $z=-1$.

\section{2 "Double scaling limit" at large $N$ in two dimensions.}

At infinite $N$ the logarithmic derivative of $Q_{N}$ is a two dimensional electric field associated with a static charge distribution representing the eigenvalues of $W$; for one realization of $W$ each eigenvalue is counted as a unit charge located at the value of the eigenvalue. When there is no gap in the eigenvalue distribution there is a jump in the field at -1 on the unit circle. When there is a gap there is no jump. The jump gets smoothed out at finite $N$ in a universal manner.

In terms of the characteristic polynomial one captures the smoothing of singularities by going to a special limit, in which two new parameters are scaled by $N^{v_{i}}$ and kept fixed as $N$ goes to infinity. The region around $z=-1$ and $t=t_{c}=4$ gets infinitely amplified. We restrict our attention to the real $z$ axis. One introduces the variables $\xi$ and $\alpha$ :

$$
z=-e^{y}, \frac{1}{t}=\frac{1}{4}+a
$$

with

$$
y=\frac{2}{6^{1 / 4} N^{3 / 4}} \xi, \quad a=\frac{1}{4 \sqrt{3 N}} \alpha
$$

We now take $N$ to infinity keeping the variables $\xi$ and $\alpha$ at fixed finite values. The numerical prefactors are chosen to make the limit of $Q_{N}(z, t)$ simple. Up to non-universal factors, $Q_{N}(z, t)$ approaches a function $\zeta(\alpha, \xi)$ :

$$
\zeta(\alpha, \xi) \equiv \int d u e^{-u^{4}-\alpha u^{2}+\xi u}
$$




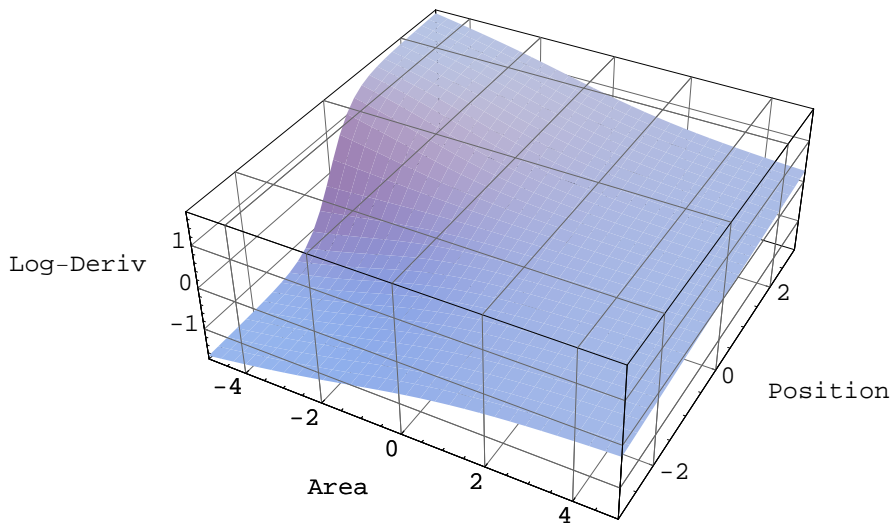

Figure 2: Plot of the logarithmic derivative of $\zeta$ with respect to $\xi$ for different values of the "area" $(\alpha)$ and "position" $(\xi)$. One sees the smooth remnant of the singularity at $\xi=0$ and its dependence on the area.

$\partial \log \zeta(\alpha, \xi) / \partial \xi$ is similar to a two dimensional electric field and its discontinuity across a charged line is smoothed as the line charge density gets depleted in the limit. Figure 2 shows the smooth result.

The meaning of large $N$ universality is that an evaluation of $Q_{N}(z, W(t))$ also in three or four dimensional $S U(N)$ pure Yang-Mills theory, where $t$ labels a dilation parameter and the shape of the loop is held fixed, would asymptote to $\zeta(\alpha, \xi)$ with the parameters $\alpha$ and $\xi$ defined from $y$ and $t-t_{c}$ using $N^{1 / 2}$ and $N^{3 / 4}$ multiplied by some finite numerical amplitudes dependent on the theory and on the shape of the Wilson loop. The essence of the claim is that the asymptotic behavior will be describable by only two parameters and that the exponents associated with the factors of $N$ relating $\alpha, \xi$ to $t, y$ will be exactly $v_{1,2}=1 / 2,3 / 4$.

\subsection{Convergence to infinite $N$ in two dimensions.}

To test this claim in simulation we first need an indication for how high $N$ ought to be for the universal behavior to set in. We plot two cross-sections through the surface of Figure 2 and check how rapidly they are approached as $N$ increases in two dimensions, where we have exact formulae also at finite $N$. The result is shown in Figures 3 and 4, for a Wilson loop that at infinite $N$ would have a gap and one that would not. For large loops the limit is approached slowly.

\section{Parameter extraction by nonlinear three parameter fits.}

In view of the slow approach to the asymptotic form, we ask how least-square best fits of it 
Convergence to scaling function with increasing $\mathrm{N}$ $\mathrm{d} / \mathrm{dy} \log \{<\operatorname{det}[\exp (\mathrm{y} / 2)+\exp (-\mathrm{y} / 2) \mathrm{W}]>\}--\mathrm{W}=$ Small loop

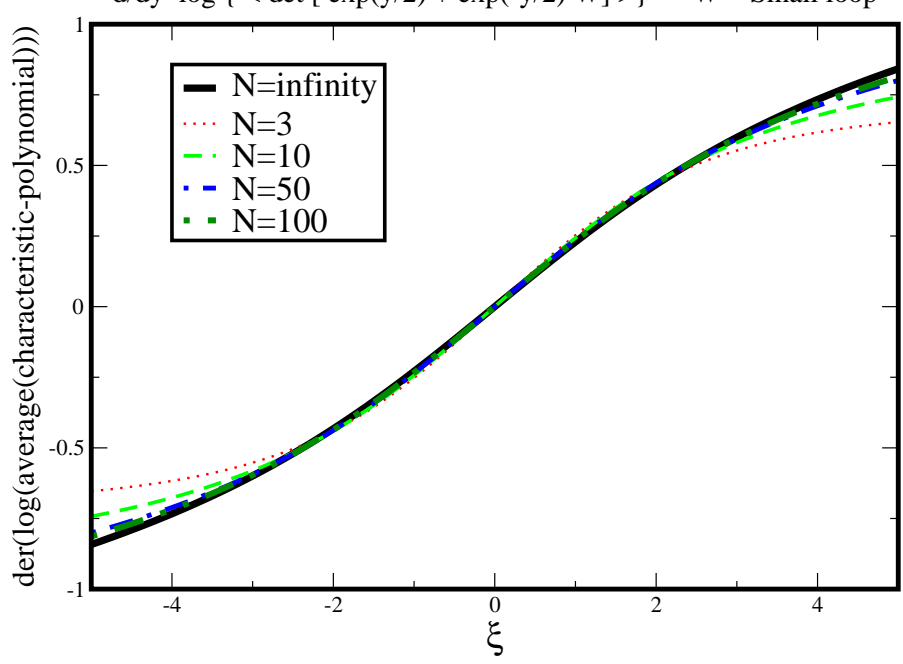

Figure 3: Wilson loop with a gap.

Convergence to scaling function with increasing $\mathrm{N}$

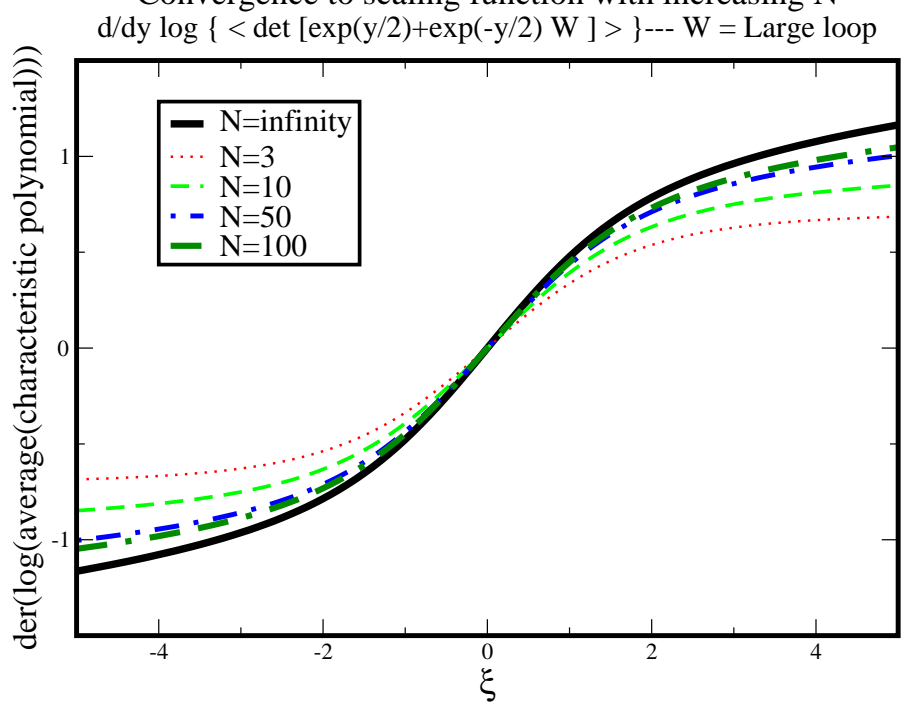

Figure 4: Wilson loop without a gap. 


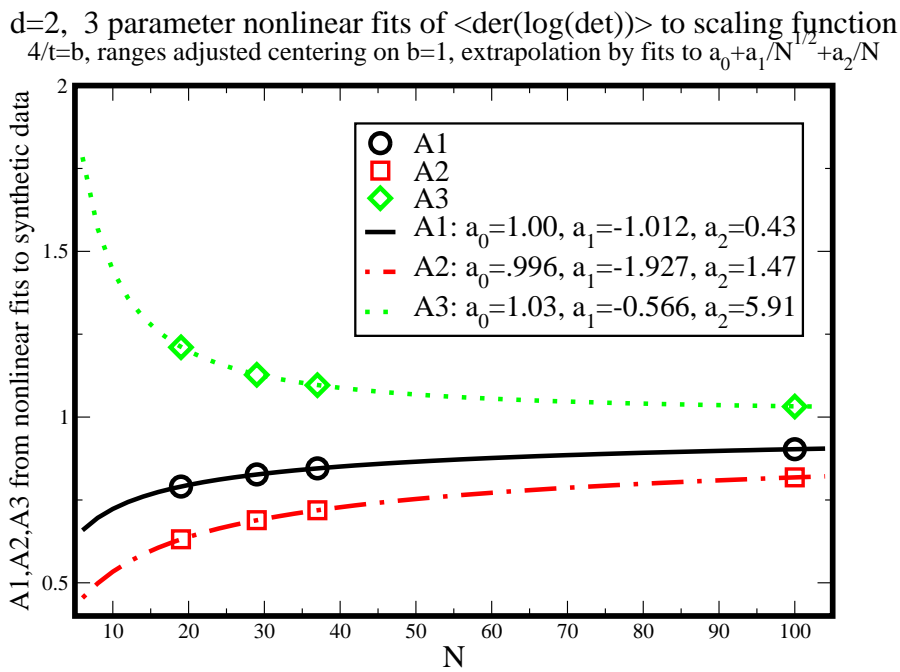

Figure 5: Analysis of synthetic two dimensional data.

to finite $N$ expressions in two dimensions would approximate the values of the critical scale $t_{c}$ and the two amplitudes relating $y$ and $t-t_{c}$ to $\xi$ and $\alpha$, when we assume $v_{i}$ to be fixed at $1 / 2$ and $3 / 4$. The parameter corresponding to $t_{c}$ is denoted by $A 3$ and the numerical coefficients multiplying $N^{v_{i}}$ entering $\alpha$ and $\xi$ are denoted by $A 2$ and by $A 1$ respectively. In two dimensions the correct values are $A 1=A 2=A 3=1$.

\subsection{Two dimensions.}

Figure 5 shows the result of doing global least square fits to $\partial \log \zeta(\alpha, \xi) / \partial \xi$ of synthetic data obtained from the exact solution in two dimensions. As indicated in the figure, one extrapolates to infinite $N$ by fitting to a series in $1 / \sqrt{N}$ - this form is a consequence of $v_{1,2}=1 / 2,3 / 4$.

This exercise shows that one may get close to the right answer even from values of $N$ that are practical, but for accuracy at the level of a fraction of a percent one would need to go to $N=100$. This value is too high for practical Monte Carlo simulations in three or four dimensions. However, $N \sim 50$ is practical and sufficient for few percent accuracies.

\subsection{Three dimensions.}

We now turn to three dimensions and carry out test runs at some convenient point. Figure 6 shows the results. We choose a case where the critical Wilson loop size occurs at an inverse 't Hooft coupling $b$ close to unity, so we can just use definitions identical to those in our analysis of the synthetic two dimensional data by identifying the parameter $t$ there with $\frac{4}{b}$ here. This is true only to 10 percent, but is enough to give us a feel for the order of magnitude of the non-universal amplitudes $A 1, A 2$ in three dimensions. 


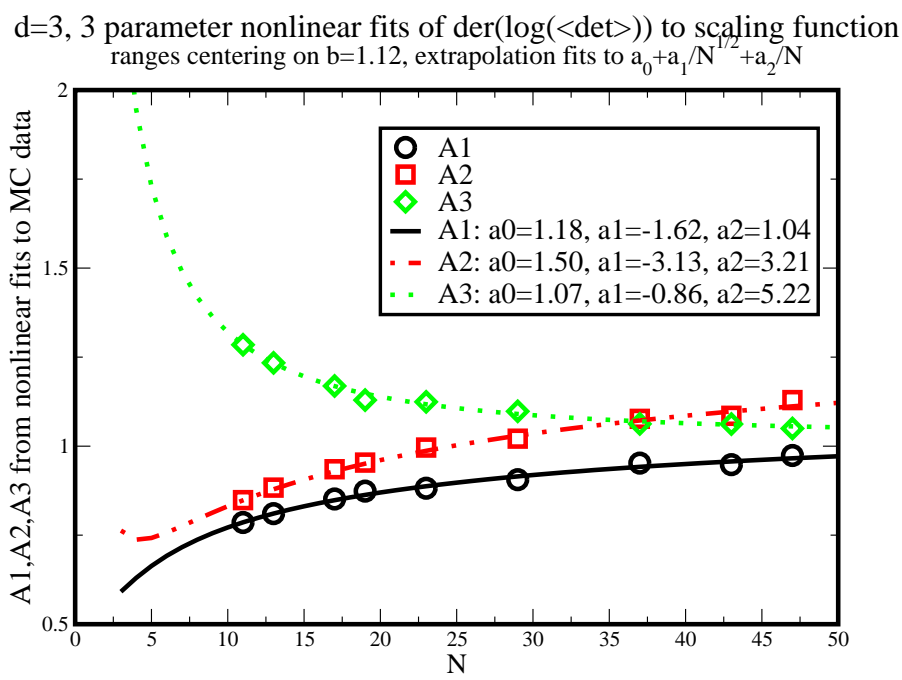

Figure 6: Analysis of three dimensional Monte Carlo data.

Unlike in two dimensions, the data has statistical errors and this further impacts accuracy. Nevertheless, one obtains an indication that universality holds with amplitudes numerically quite close to their values in two dimensions. This is accidental to some degree and cannot hold in general, as in three dimensions there are Wilson loop of shapes unattainable in two dimensions.

\section{Conclusions.}

We conclude that it should be possible to check our main claim in three dimensions but the method of analysis ought to be improved. So far, the numerical results in 3D are in agreement

with large $N$ universality. Amplitudes relating to simple square Wilson loops in three dimensions come out surprisingly close to their two dimensional values; perhaps, this is a reflection of the approximate dimensional reduction known to hold since the 80's.

\section{Acknowledgments}

R.N. acknowledges partial support by the NSF under grant number PHY-055375. H. N. acknowledges partial support by the DOE under grant number DE-FG02-01ER41165.

\section{References}

[1] R. Narayanan and H. Neuberger, JHEP 0603 (2006) 064.

[2] R. A. Janik, W. Wieczorek, J. Phys. A: Math. Gen. 37, (2004) 6521.

[3] B. Durhuus and P. Olesen, Nucl. Phys. B 184 (1981) 461. 